\title{
CONSTRUÇÃO DE UM INSTRUMENTO DE PASSAGEM DE PLANTÃO
}

Tânia Roberta Limeira Felipe'. Wilza Carla Spiri²

Objetivo: descrever a construção de um instrumento de passagem de plantão utilizando a metodologia SBAR (Situation-BackgroundAssessment-Recommendation). Método: trata-se de estudo metodológico para construção de um instrumento de passagem de plantão em uma enfermaria de gastroenterologia cirúrgica. Foi realizada uma revisão bibliográfica abrangente sobre a metodologia SBAR. O estudo foi aprovado pelo Comitê de Ética em Pesquisa da instituição cenário da pesquisa. Resultados: os itens constantes do instrumento foram: dados de identificação do paciente que incluíram: data, leito, nome, idade sexo, data de admissão; indicadores: sistema de classificação de paciente, presença de acompanhante; Situação (S): diagnóstico médico e diagnósticos de enfermagem; Breve história (B): alergias, co-morbidades, histórico cirúrgico, isolamento/precauções e barreiras para a comunicação; Avaliação (A): sinais vitais, alimentação, eliminações, curativos, drenos, cateteres e medicamentos; Recomendação (R): interconsultas, intervenções de enfermagem e intercorrências. Conclusão: $\mathrm{O}$ instrumento contribui para a padronização da passagem de plantão dos profissionais de enfermagem.

Descritores: Gerenciamento da prática profissional, Estudos de validação, Sistema de comunicação no hospital, Equipe de enfermagem.

\section{CONSTRUCTION OF A SHIFT CHANGE INSTRUMENT}

Objective: to describe the construction of a shift change instrument using the SBAR (Situation-Background-Assessment-Recommendation) methodology. Methodolgy: this is a methodological study for the construction of an instrument of a shift change in a surgical gastroenterology ward. A comprehensive bibliographic review was done on the SBAR methodology. The Research Ethics Committee of the scenario research institution approved the study. Results: the items included in the instrument were: patient identification data that included: date, bed, name, age, sex, date of admission; indicators: patient classification system, presence of companion; Situation (S): medical diagnosis and nursing diagnoses; Background (B): allergies, comorbidities, surgical history, isolation / precautions and barriers to communication; Assessment (A): vital signs, nutritional aspects, eliminations, dressings, drains, catheters and medications; Recommendation (R): interconsultations, nursing interventions and intercurrences. Conclusion: The instrument contributes to the standardization of nurses' change of shift.

Descriptors: Practice management, Validation studies, Hospital communication systems, Nursing team.

\section{CONSTRUCCIÓN DE UN INSTRUMENTO DE PASO DE PLANTÓN}

Objetivo: describir la construcción de un instrumento de paso de turno utilizando la metodología SBAR (Situation-Background-AssessmentRecommendation). Método: se trata de un estudio metodológico para la construcción de un instrumento de paso de turno en una enfermería de gastroenterología quirúrgica. Se realizó una revisión bibliográfica exhaustiva sobre la metodología SBAR. El estudio fue aprobado por el Comité de Ética en Investigación de la institución escenario de la investigación. Resultados: los ítems constantes del instrumento fueron: datos de identificación del paciente que incluyeron: fecha, lecho, nombre, edad sexo, fecha de admisión; indicadores: sistema de clasificación de pacientes, presencia de acompañante; Situación (S): diagnóstico médico y diagnósticos de enfermeria; Breve historia (B): alergias, comorbilidades, histórico quirúrgico, aislamiento / precauciones y barreras para la comunicación; Evaluación (A): signos vitales, alimentación, eliminaciones, curativos, drenajes, catéteres y medicamentos; Recomendación (R): interconsultas, intervenciones de enfermería e intercurrencias. Conclusión: El instrumento contribuye a la padronización de los cambios de turno de los profesionales de enfermería. Descriptores: Gestión de la práctica profesional, Estudios de validación, Sistemas de comunicación en hospital, Grupo de enfermeira.

1Hospital das Clinicas da Faculdade de Medicina de Botucatu - UNESP

${ }^{2}$ Departamento de Enfermagem da Faculdade de Medicina de Botucatu -UNESP

Autor correspondente: Wilza Carla Spiri. E-mail: wilza.spiri@unesp.br 


\section{INTRODUÇÃO}

A enfermagem tem como premissa essencial - cuidado, e para realizá-lo com qualidade faz-se necessário utilizar instrumentos em seu processo de trabalho que possibilitem a articulação da teoria com a prática.

Dentre as atividades fundamentais para o trabalho na Enfermagem, a passagem de plantão é um dos elementos cruciais, pois é um processo que se refere a informação específica de pacientes que passam de um profissional de saúde para outro, ou de uma equipe de profissionais assistenciais para outra equipe ou ainda de profissionais de saúde para pacientes e familiares quando esses vão para a casa(1).

No processo de trabalho da enfermagem, a passagem de plantão possibilita a troca de informações entre a equipe que prestou assistência com a equipe que assumirá os cuidados no turno seguinte e a visão geral sobre o cenário, além de diagnosticar, planejar, delegar e aplicar as intervenções necessárias ${ }^{(2,3)}$.

Algumas estratégias devem ser consideradas na passagem de plantão: 1) assegurar que a organização de saúde implemente, de forma padronizada, a comunicação na passagem de plantão sugerindo o uso da técnica SBAR para essa comunicação; considerar a alocação de tempo suficiente para que as informações importantes sejam comunicadas e a não interrupção durante esse processo; informações referentes a condição do paciente, medicamentos, planos de tratamento e mudanças na condição do paciente são essenciais; 2) assegurar que organizações de saúde implementem sistemas que garantam a alta do paciente com todas as informações necessárias ao seu tratamento, como por exemplo, diagnóstico, plano de tratamento, medicamentos e resultados de exames; 3) incorporar treinamento na comunicação da passagem de plantão de forma permanente; 4) encorajar a comunicação entre a organização de saúde e os prestadores de cuidado (formal e informal) $)^{(1)}$

Cada instituição e cada profissional têm sua dinâmica para realizar a passagem de plantão. 0 comprometimento e a valorização dessa ação devem ficar claras ao profissional, para que a transmissão da informação seja produtiva e eficaz, independentemente do método que se utiliza ou a unidade de prestação de serviços $^{(4)}$

No entanto, fatores como quantidade excessiva ou reduzida de informações, pouca possibilidade de realizar questionamentos durante o processo, informações inconsistentes, omissão ou informações erradas, não existência de processos padronizados, registros falhos e ilegíveis, interrupções e distrações, além de uma equipe não preparada para esse processo, são aspectos que dificultam essa prática com prejuizo para o paciente ${ }^{(5)}$

O Código de Ética dos Profissionais de Enfermagem explicita a legalidade do processo de passagem de plantão, pois no artigo 38 destaca como dever do profissional " Prestar informações escritas e/ou verbais, completas e fidedignas, necessárias à continuidade da assistência e segurança do paciente"(6).

O SBAR (Situation-Background-AssessmentRecommendation) é uma ferramenta de comunicação que consiste no preenchimento de quatro critérios: S: Situação, B: Breve Histórico, A: Avaliação e R: Recomendação, permitindo a organização da informação que está sendo emitida, tornando-a clara e padronizada, de forma que as informações essenciais do cuidado com paciente não se percam durante a troca de informações ${ }^{(7)}$. O uso da ferramenta SBAR possibilita o maior foco no paciente, diálogo entre a equipe mais efetivo e menor transcrição de dados. Assim, a ferramenta demonstrou ser concisa, consistente, compreensiva e com o relato focado no paciente ${ }^{(8)}$.

$\mathrm{Na}$ enfermagem, a utilização da ferramenta SBAR para a passagem de plantão é mais recente, especialmente no Brasil, e tem evidenciado tratarse de uma comunicação efetiva, pois proporciona segurança para a equipe de enfermagem pela acurácia das informações, além de ser simples e contribuir para a segurança do paciente ${ }^{(9)}$

Este estudo tem como finalidade construir um instrumento de passagem de plantão com base na ferramenta recomendada pela Organização Mundial de Saúde, o SBAR, pois acredita-se ser esse instrumento necessário para melhorar a qualidade da informação transmitida entre os turnos e até mesmo entre os setores, como, por exemplo, na transferência de pacientes, resultando na continuidade da assistência.

\section{OBJETIVO}

Construir um instrumento de passagem de plantão utilizando a metodologia SBAR.

\section{MÉTODO}

\section{Tipo de Estudo}

Trata-se de estudo metodológico cujo objetivo é trabalhar com instrumentos e ferramentas complexas e desenvolvimento de referenciais metodológicos ${ }^{(10)}$. 


\section{Local do Estudo}

O instrumento foi construído considerando o cenário de assistência de uma enfermaria de Gastroenterologia cirúrgica de um hospital público de ensino do Estado de São Paulo.

\section{Procedimentos de coleta e análise dos dados}

O instrumento foi construido no período de setembro a novembro de 2018, após revisão da literatura sobre passagem de plantão e a metodologia SBAR e das características da enfermaria de Gastroenterologia cirúrgica do hospital.

O método para revisão da literatura foi a revisão integrativa considerando as etapas de identificação do problema; seleção da amostra; definição das características da pesquisa; avaliação/análise dos estudos incluidos na revisão; discussão e interpretação dos resultados e apresentação da revisão/síntese do conhecimento ${ }^{(11)}$

A pergunta norteadora foi: qual foi o conhecimento produzido e publicado sobre a passagem de plantão dos profissionais de enfermagem por meio da ferramenta SBAR?

A busca dos artigos e a seleção da amostra ocorreram em julho de 2018, e o instrumento principal utilizado foi a busca na Biblioteca Virtual de Saúde (BVS) com os descritores: passagem de plantão AND enfermagem AND SBAR para os artigos brasileiros e change of shift OR handover AND nursing AND SBAR para a busca dos artigos internacionais. Com a delimitação dos descritores, foi possivel selecionar a amostra inicial da pesquisa, que adotou os seguintes critérios de inclusão: artigos na íntegra, encontrados nas literaturas nacional e internacional, nas línguas portuguesa, inlgesa e espanhola, publicados no período de janeiro de 2008 a julho de 2018.

Com os descritores em português apenas um artigo foi encontrado e com os descritores em Inglês foram encontrados sete artigos.

Após a seleção dos artigos, realizou-se leitura criteriosa do título e do resumo e, em seguida, foram excluídos aqueles que não respondiam à pergunta norteadora.

\section{Procedimentos Éticos}

O instrumento, objeto dessa investigação, é a primeira etapa de uma dissertação de Mestrado Profissional em Enfermagem aprovada pelo Comitê de Ética em Pesquisa -parecer 3.009.068 e CAAE 02168918.0.0000.5411.

\section{Resultados}

Da leitura sistemática sobre o objeto abordado, obteve-se uma amostra final de seia artigos científicos, pois dois deles não se enquandraram nos critérios de inclusão.

Assim, o instrumento foi construido com base na literatura sobre o SBAR e na especificidade da enfermaria da gastroenterologia cirúrgica. Os itens constantes do instrumento abrangeram aspectos considerados essenciais para uma comunicação efetiva entre os membros da equipe de enfermagem.

Foram construidos com os seguintes elementos: dados de identificação, indicadores, situação (S), breve história (B), avaliação (A) e recomendação (R).

Em dados de identificação do paciente, foram incluídos: unidade de internação, data da passagem de plantão, data de admissão do paciente, número do leito, nome do paciente, data de nascimento/idade e sexo. Em indicadores, incluíram-se quedas, lesão por pressão, TEV (tromboembolismo venoso periférico), SCP (sistema de classificação de pacientes), presença ou não de pulseira de identificação do paciente e a presença ou não de acompanhantes.

No item Situação (S) foram descritos o dia da internação, diagnóstico médico, diagnósticos de enfermagem ou levantamento de problemas de enfermagem nas últimas 24 horas e o espaço para identificação do profissional de enfermagem responsável pela coleta dos dados. No item Breve história (B), os elementos propostos foram: alergias, co-morbidades, histórico cirúrgico, isolamento/precauções e barreiras para a comunicação.

Em Avaliação (A), foram incluidos: sinais vitais, oxigenação/ventilação, consciência, locomoção, presença de drenos, cateteres, sondas, realização de exames, aspectos nutricionais, curativos, eliminações, medicamentos nas diferentes vias e intercorrências apresentadas durante o plantão. No item Recomendação (R), os elementos foram: interconsultas, intervenções de enfermagem e outros dados necessários.

Todas as siglas utilizadas no instrumento são de conhecimento dos membros da equipe na unidade de gastroenterologia cirúrgica, cenário da pesquisa; também, foi mantido um glossário nessa unidade. Esses itens visaram abranger elementos essenciais para a passagem de plantão, possibilitando a continuidade da assistência de enfermagem. Para viabilidade da utilização do instrumento, todos os itens foram sintetizados em apenas uma página. O quadro 1 apresenta o instrumento construido: 
Instrumento de Passagem De Plantão - Sbar

Unidade de Internação:

Nome do Paciente:

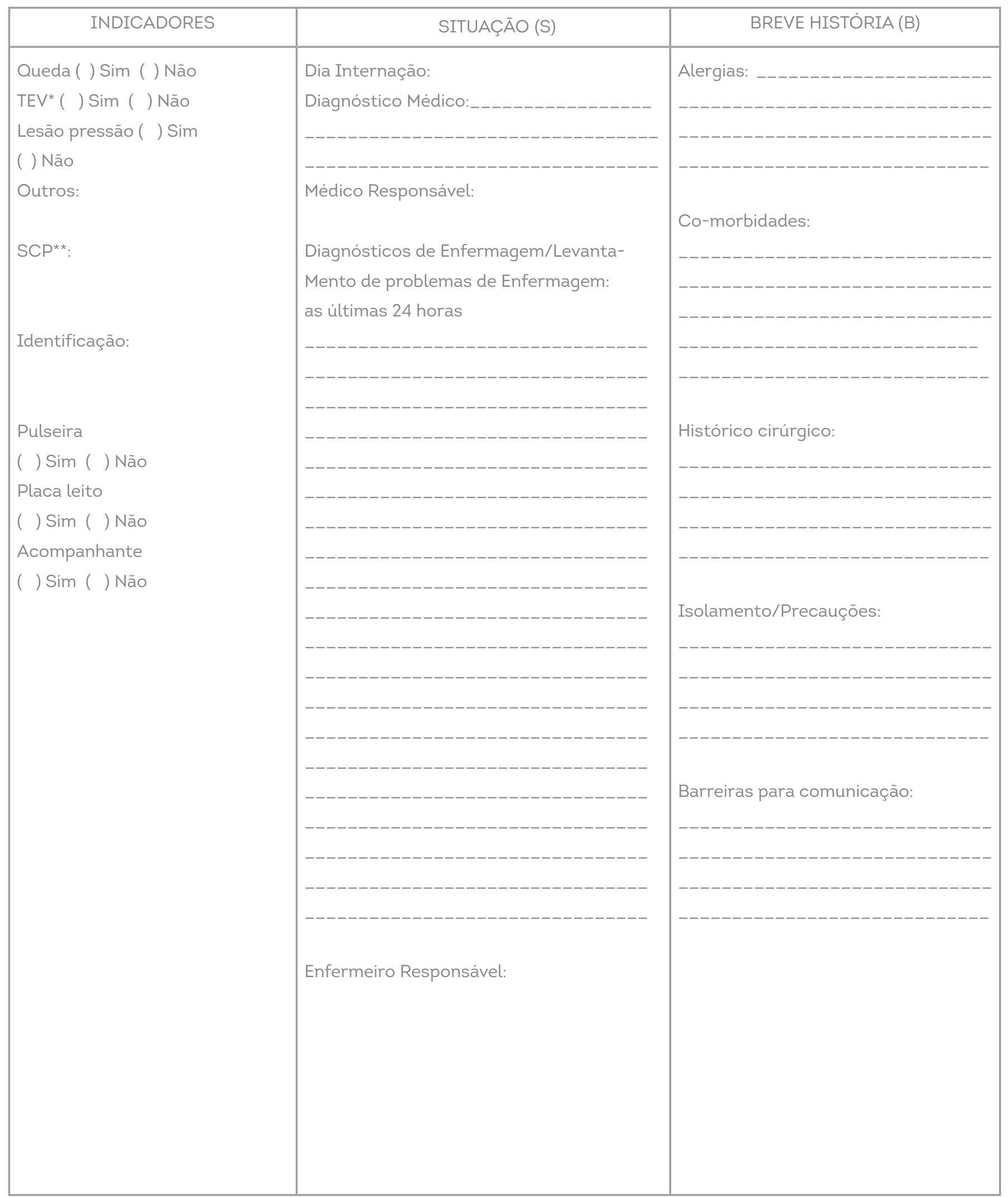


Data:

Leito:

Sexo: $M() F(~)$
Data de Admissão:

Dn/Idade:

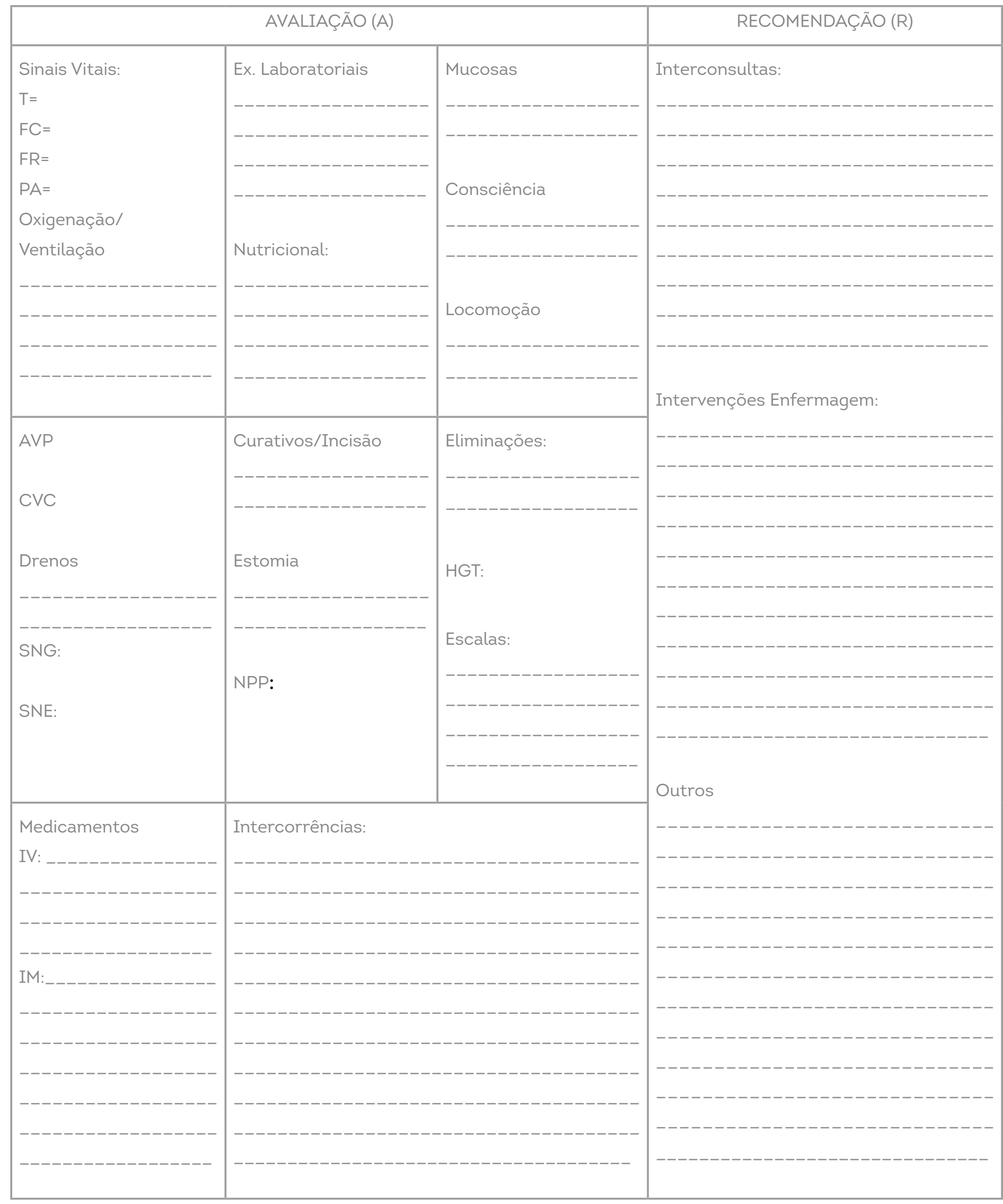




\section{DISCUSSÃO}

O presente estudo teve como objetivo principal descrever o processo de construção de um instrumento de passagem de plantão usando a metodologia SBAR, disponibilizando um impresso prático e fácil de usar, auxiliando profissionais que utilizam a passagem de plantão como instrumento de trabalho diário; uma ferramenta que visa auxiliar e melhorar a comunicação entre as equipes de saúde.

Ressalta-se que a passagem de plantão na enfermaria, cenário desse estudo, acontecia em forma de reunião. Algumas informações consideradas relevantes pela equipe eram anotadas em um papel "rascunho", cuja inutilização ocorria logo após a troca do plantão; por vezes, alguns profissionais optavam apenas por uma passagem de plantão de forma oral.

A construção do instrumento atendeu a proposição da Organização Mundial da Saúde que refere ser importante existir uma forma padronizada de passagem de plantão, sugerindo o uso da técnica SBAR para essa comunicação(1). Destaca-se que as informações entre a equipe de enfermagem sejam de forma objetiva e concisa, abordando os acontecimentos referentes à assistência direta e/ou indireta ao paciente, durante o turno de trabalho, assim como assuntos de interesse institucional ${ }^{(12)}$

Estudo destaca que quando há quebra na continuidade do tratamento e na qualidade da assistência decorrente da má comunicação, evidenciam-se prejuízos relevantes, abrindo uma lacuna entre a qualidade e a assistência(13). A utilização da ferramenta SBAR pode contribuir para que os erros de comunicação diminuam e fatores contribuintes da melhoria de atitudes de segurança sejam incrementados, pois é uma forma padronizada de passagem de plantão que é válida para a comunicação entre a equipe de saúde ${ }^{(14)}$.

A inclusão de itens de acordo com a realidade da enfermaria de gastroenterologia cirúrgica será importante para a adesão dos profissionais de enfermagem na passagem de plantão.

Estudo destaca que o histórico, medicamentos em uso, alergias, resultado de exames relevantes, sinais vitais, impressões clínicas, alterações de exame físico, presença de dispositivos, precauções, exames complementares e específico, além de outras recomendações, são dados importantes na passagem de plantão(15).

A construção da ferramenta SBAR em uma maternidade compreendeu as fases: Situação (Situation), descrição concisa do motivo de internação, tipo de parto e data atual. A segunda, Quadro Atual (Background), englobou os critérios do quadro atual das puérperas e recém-nascidos compreendendo aspectos gerais como o nível de consciência, necessidade de restrição no leito, tipo de dieta e alimentação, necessidade de dispositivo para oxigenação, presença de acesso venoso e específicos como a produção de colestro, característica da mama, avaliação de lóquios, eliminações fisiológicas, dentre outros. A Avaliação Profissional (Assessment), local para descrição da opinião do profissional sobre o contexto da puérpera e recém-nascido, abordando gravidade e intercorrências, e a quarta e última etapa caracterizou as Recomendações (Recommendation), isto é, o que pode ser feito para corrigir os problemas e a descrição das pendências, recomendações à puérpera e recém nascido( ${ }^{(16)}$. Evidenciando assim a necessidade de construir a ferramenta que melhor se adéque ao contexto de sua utilização.

Estudo evidencia que técnicas de comunicação estruturadas como o SBAR melhoram a percepção, entre os membros da equipe de saúde, do processo de passagem de plantão e da colaboração necessária para esse processo, especialmente quando o conteúdo dessa passagem de plantão tem coerência com as necessidades dos pacientes assistidos $^{(17)}$

Estudos conduzidos nos Estados Unidos da América demonstraram que o uso da ferramenta SBAR para passagem de plantão da equipe de enfermagem promoveu melhor estrutura, consistência, priorização, acurácia e compreensão das informações necessárias ao cuidado. Além disso, o uso da técnica possibilitou melhor comunicação e conhecimento sobre os pacientes assistidos ${ }^{(7.8)}$.

A construção de ferramentas para passagem de plantão em formato de checklist proporciona o planejamento do cuidado seguro ao paciente, pois contempla informações que contemplam nome do paciente, registro, data, história prévia, cirurgia, tipo de anestesia, dentre outros dados essenciais para conhecer a situação do paciente, sendo a sua objetividade um facilitador à implementação pelos profissionais ${ }^{(18)}$.

Estudo realizado em Unidade de Terapia Intensiva corrobora essa pesquisa, pois evidenciou a necessidade de construção de instrumentos de passagem de plantão com conteúdo apropriado a essa unidade auxiliando na transmissão de informações durante esse processo e contribuindo para garantir a segurança do paciente minimizando os riscos e falhas na comunicação(19).

Estudo que teve como objetivo implementar uma ferramenta de passagem de plantão por meio do acrônimo ISBAR (Identification, Situation, Background, Assessment \& Action, Response/Rationale) em uma unidade de pronto atendimento concluiu que é crucial utilizar uma ferramenta padrão, formal e sistematizada para garantir que a transferência dos cuidados seja "eficaz, completa e objetiva" com possibilidade da segurança do paciente e equipe ${ }^{(20)}$.

\section{LIMITAÇÕES DO ESTUDO}

Como limitação do estudo, tem-se que o instrumento necessita ser validado para que possa ser colocado na prática. Assim, as etapas posteriores do estudo são a validação por meio de juízes e sua aplicação pelos membros da equipe de enfermagem no cenário para o qual o instrumento foi construído. 


\section{Contribuições do estudo para a prática}

A existência de um instrumento para a passagem de plantão por meio da ferramenta SBAR possibilitará que os profissionais de enfermagem realizem essa importante etapa de seu processo de trabalho de forma padronizada contribuindo para que as informações garantam a continuidade do cuidado e a segurança do paciente e da própria equipe.

\section{CONCLUSÃO}

Construiu-se um instrumento para passagem de plantão na enfermaria de gastroenterologia cirúrgica de um hospital de ensino no Estado de São Paulo utilizando-se a ferramenta SBAR, considerando dados de identificação do paciente; indicadores; Situação (S) (dia da internação, diagnóstico médico, diagnósticos de enfermagem ou levantamento de problemas de enfermagem nas últimas 24 horas); Breve história (B) (alergias, co-morbidades, histórico cirúrgico, isolamento/precauções e barreiras para a comunicação); Avaliação (A), (sinais vitais, oxigenação/ventilação, consciência, locomoção, drenos, cateteres, sondas, exames, aspectos nutricionais, curativos, eliminações, medicamentos e intercorrências); Recomendação (R) (interconsultas, intervenções de enfermagem e outros dados necessários).

O instrumento contribui para a padronização da passagem de plantão dos profissionais de enfermagem.

\section{Contribuição dos autores:}

Ambos autores participaram da: 1)concepção; 2) análise e interppretação dos dados; 3) redação do artigo; 4) revisão crítica e 5) revisão.

\section{REFERÊNCIAS}

1. World Health Organization (WHO). World alliance for patient safety. Communication during patient hand-overs. Patient safety solutions [Internet]. 2007 [cited 2019 Mar 08]. Available from: https://www.who.int/patientsafety/solutions/ patientsafety/PS-Solution3.pdf?ua=1

2. Gonçalves MI, Rocha PK, Souza S, Tomazoni A, Paz BPD, Souza AIJ. Segurança do paciente e passagem de plantão em cuidados intensivos neonatais. Rev. Baiana enferm. [Internet]. 2017 [cited 2019 Jun 24];31(2):el7053. Available from: https:// portalseer.ufba.br/index.php/enfermagem/article/view/17053/14606

3. Portal KM. Magalhães AMM. Passagem de plantão: um recurso estratégico para a continuidade do cuidado em enfermagem. Rev. Gaúcha Enferm., [Internet]. 2008 [cited 2019 Jun 21];29(2):246-53. Available from: https://seer.ufrgs.br/ RevistaGauchadeEnfermagem/article/view/5588/3198

4. Siqueira ILCP, Kurcgant P. Passagem de plantão: falando de paradigmas estratégias. Acta Paul Enferm. [Internet]. 2005 [cited 2019 Jun 21];18(4):446-51. Available from: http://www.scielo.br/pdf/ape/v18n4/al5v18n4.pdf

5. Gonçalves MI, Rocha PK, Anders JC, Kusahara DM, Tomazoni A. Comunicação e segurança do paciente na passagem de plantão em unidades de cuidados intensivos neonatais. Texto \& Contexto Enferm [Internet]. 2016 [cited 2019 Jun 24]; 25(1):e2310014. Available from: http://www.scielo.br/pdf/tce/v25nl/ 0104-0707-tce-25-01-2310014.pdf

6. Conselho Federal de Enfermagem. Resolução COFEN № 564/2017 [Internet]. 2017 [cited 2019 Mar 08]. Available from: http://www.cofen.gov.br/resolucao-cofen-no-5642017_59145.html.

7. Cornell P, Gervis MT, Yates L, Vardaman JM. Impact of SBAR on nurse shift reports and staff rounding. Med. Surg Nursing [Internet]. 2014 [cited 2019 Jun 18];23(5):334-42. Available from: https://insights.ovid.com/medsurg-nursing/mednu/2014/09/000/impact-sbar-nurse-shift-reports-staff-rounding/12/00008484

8. Cornell P, Gervis MT, Yates L, Vardaman JM. Improving shift report focus and consistency with the Situation, Background, Assessment, Recommendation protocol. JONA [Internet]. 2013 [cited 2019 Jun 18]:43(7/8):422-8. Available from: https://insights.ovid.com/article/00005110-201307000-00011

9. Stewart KR, Hand KA. SBAR, communication, and patient safety: an integrated literature review. Medsurg Nursing [Internet]. 2017 [cited 2019 Jun 18];26(5):297305. Available from: https://insights.ovid.com/medsurg-nursing/mednu/2017/09/000/sbar-communication-patient-safety-integrated/3/00008484

10. Polit D. F; Beck C. T. Fundamentos de Pesquisa em enfermagem: avaliação de evidências para as práticas da enfermagem. 7a ed. Porto Alegre (RS): Artmed: 2011. $669 p$

11. Whittemore R, Knafl K. The integrative review: update methodology. J Adv Nurs. [Internet]. 2005 [cited 2018 May 10];52(5):546-53. Available from: http://citeseerx.ist.psu.edu/viewdoc/download?doi=10.1.1.465.93938rep=repl\&type=pdf

12. Silva EE, Campos LF. Passagem de plantão na enfermagem: revisão de literatura. Cogitare Enferm [Internet]. 2007 [cited 2019 Jun 16]:12(4):502-7. Available from: https://revistas.ufpr.br/cogitare/article/view/10077/6929

13. Welsh CA., Flanagan ME, Ebright P. Barriers and facilitators to nursing handoffs: recommendations for redesign. Nursing Outlook [Internet]. 2010 [cited 2019 Jun 10]: 58(3):148-54. Available from: https://www.sciencedirect.com/science/ article/pii/SO02965540900195X?via\%3Dihub 14. Ting WH, Peng FS, Lin HH, Hsiao SM. The impact of situation-background-assessment-reommendation (SBAR) on safety attitudes in the obstetrics department. Taiwanese Journal of Obstetrics \& Gynecology [Internet]. 2017 [cited 2019 Jun 15]: 56:171-4. Available from: https:// www.researchgate.net/publication/316674002_The_impact_of_situation-background-assessment-recommendation_SBAR_on_safety_attitudes_in_the_ obstetrics_department

15. Pedro DF, Nicola AL, Oliveira JL. Passagem de plantão entre profissionais de enfermagem hospitalares: análise de fatores influentes. Rev UNINGÁ [Internet] 2016 [acesso em $2019 \mathrm{Jul}$ 15];25(1):27-31. Available from: https://www.mastereditora.com.br/periodico/20160102_215634.pdf

16. Nascimento JSG, Rodrigues RR, Pires FC, Gomes BF. Passagem de plantão como ferramenta de gestão para segurança do paciente. Rev Enferm UFSM [Internet]. 2018 [cited 2019 Jun 15]:8(2):544-59. Available from: https://periodicos. ufsm.br/reufsm/article/view/29412

17. Martin HA, Ciurzynski SM. Situation, background, assessment, and recommendation - guide huddles improve communication and teamwork in the emergency department. Journal of Emergency Nursing [Internet]. 2015 [cited 2019 Jun 15];41(6):484-8. Available from: https://reader.elsevier.com/ reader/sd/pii/s0099176715002287?token=0C093CB778E4A510A7D9CC11106AF32516B2563A04C449E3B02EEB2910B1FD7C386294BFB406B8D9472D700A959D82B6

18. Silva SG, Nascimento ERP. Hermida PMV, Sena AC, Klein TCR, Pinho FM. Checklist para passagem de plantão de pacientes em pós-operatório imediato na admissão em terapia intensiva. Enferm Foco [Internet]. 2016 [cited 2019 Jul 11]:7(1):13-17. Available from: http://revista.cofen.gov.br/index.php/enfermagem/ article/view/658/277

19. Corpolato RC, Mantovani MF, Willig MH, Andrade LAS de, Mattei AT, Arthur JP. Padronização da passagem de plantão em Unidade de Terapia Intensiva Geral Adulto. Rev. Bras. Enferm. [Internet]. 2019 [cited 2019 Jul 11];72(Supp 1):88-95. Available from: http://www.scielo.br/scielo.php?script=sci_arttext8pi$\mathrm{d}=$ S0034-71672019000700088\&lng=pt. $\mathrm{http}: / / \mathrm{dx}$.doi.org/10.1590/0034-71672017-0745

20. Silva DA, Rocha IMS, Dias FA, Moreira DA, Afonso LN, Brito MJM. Otimização da ferramenta utilizada durante a passagem de plantão em uma unidade de pronto atendimento. SANARE [Internet]. 2017 [cited 2019 Jul 11];16(1):118-23. Available from: https://sanare.emnuvens.com.br/sanare/article/view/1102

RECEBIDO EM: 24/06/2019. - ACEITO EM: 06/09/2019

82 Enferm. Foco 2019: 76-82 